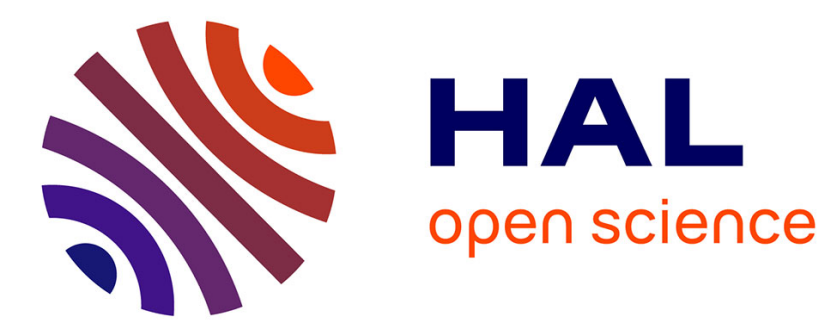

\title{
Un nouvel instrument de travail pour la communauté scientifique: le répertoire des collégiales séculières de France à l'époque médiévale
}

Anne Massoni

\section{- To cite this version:}

Anne Massoni. Un nouvel instrument de travail pour la communauté scientifique: le répertoire des collégiales séculières de France à l'époque médiévale. Revue d'Histoire ecclésiastique , 2007, 3-4 (102), pp.915-939. hal-01156440

\section{HAL Id: hal-01156440 \\ https://hal-unilim.archives-ouvertes.fr/hal-01156440}

Submitted on 17 Dec 2017

HAL is a multi-disciplinary open access archive for the deposit and dissemination of scientific research documents, whether they are published or not. The documents may come from teaching and research institutions in France or abroad, or from public or private research centers.
L'archive ouverte pluridisciplinaire HAL, est destinée au dépôt et à la diffusion de documents scientifiques de niveau recherche, publiés ou non, émanant des établissements d'enseignement et de recherche français ou étrangers, des laboratoires publics ou privés. 


\section{UN NOUVEL INSTRUMENT DE TRAVAIL POUR LA COMMUNAUTE SCIENTIFIQUE: LE REPERTOIRE DES COLLEGIALES SECULIERES DE FRANCE A L'EPOQUE MEDIEVALE (1)}

L'article présenté ici vise à rendre compte du travail entrepris depuis quelques années par plusieurs chercheurs, périodiquement réunis grâce à leurs études sur les collégiales françaises, et qui ont pris plaisir à regrouper et recouper leurs connaissances pour donner naissance à un nouvel instrument de travail dont on espère qu'il sera utile à une plus large communauté scientifique.

\section{Bilan historiographique}

Le lancement des études canoniales a déjà plus d'un demi-siècle derrière lui alors qu'à la différence des moines, les chanoines furent longtemps les « oubliés de l'histoire de l'Église ». On le doit à quelques publications pionnières qui datent des années $1950\left(^{2}\right)$. Depuis, ces travaux ont suivi deux principales directions de recherche dans l'historiographie française. La première concerne les chanoines réguliers et la seconde les chanoines séculiers des cathédrales. Le travail accompli dans le domaine des réguliers l'a surtout été dans la perspective d'une histoire de la spiritualité, même si cette dernière s'asortit d'études sur les établissements classés par ordres canoniaux, comme c'est le cas dans l'oeuvre majeure de dom Becquet $\left({ }^{3}\right)$. Le récent colloque organisé par le CERCOR au Puy du 29 juin au $1^{\text {er }}$ juillet 2006 a débuté par un hommage à l'oeuvre du moine de Ligugé puis a proposé des communications qui vont dans le sens de synthèses «nationales » (France, Empire, espace flamand, îles britanniques, Italie, péninsule ibérique, Terre sainte).

À la fin des années 1980, une autre rencontre scientifique tenta d'élargir l'angle de vue à propos d'histoire canoniale, ce qu'annonçait son titre: Le monde des chanoines $\left({ }^{4}\right)$. Son contenu resta cependant encore résolument orienté sur les chapitres collégiaux et les ordres réguliers. Il offrit aussi des ouvertures sur les cathédrales et les chanoines en général, mais sans distinction particulière pour les chanoines séculiers, et encore moins les chanoines de collégiales. Au mieux s'intéresse-t-on à leur régularisation. Les propos liminaires rédigés par M.-H. Vicaire $\left({ }^{5}\right)$ exposent la

1 Je remercie très chaleureusement monsieur Vincent Tabbagh, professeur d'histoire du Moyen Âge à l'Université de Bourgogne, pour la relecture attentive de ce texte et ses remarques toujours judicieuses.

2 Comme l'article «chanoines » du Dictionnaire d'histoire et de géographie ecclésiastiques 12 (1953), col. 353405 que l'on doit à Ch. DEREINE qui y annonce d'emblée que dans les domaines économique, social, politique et intellectuel, le rôle des chapitres fut comparable à celui des monastères jusqu'à la fin de l'Ancien Régime. Il examine l'histoire canoniale en quatre phases: origines, époque mérovingienne, époque carolingienne, réforme grégorienne et s'arrête significativement à l'époque de la réforme. Suit une rencontre déterminante sur ce sujet, qui fait encore largement autorité, la première semaine d'études de la Mendola en 1959: La vita commune del clero nei secoli XI e XII. Atti della Settimana di Studi Mendola, settembre 1959, 2 vol., Milan, 1962. Et particulièrement pour le domaine qui nous intéresse: J.-Fr. LEMARIGNIER, Aspects politiques des fondations de collégiales dans le royaume de France au XI siècle, p. 19-49.

3 Qui porte surtout sur les communautés de chanoines augustins du Limousin et particulièrement sur les ordres d'Aureil et de l'Artige mais qui s'attela aussi à des synthèses plus larges sur les réalités aquitaines et françaises. Ses principaux articles ont été réunis dans J. BECQUET, Vie canoniale en France aux $X^{e}$-XII ${ }^{e}$ siècles, Londres, Variorum Reprints, 1985, 292 p. Dom Becquet récapitule lui-même la bibliographie générale dans Vingt-cinq années d'études canoniales en France (1959-1984), dans Liber amicorum. Études historiques offertes à Pierre Bougard, Arras, 1987, p. 65-77. On doit se reporter aujourd'hui au bilan historiographique proposé dans A. VAUCHEZ (dir.), C. CABY, L'histoire des moines, chanoines et religieux au Moyen Âge, guide de recherche et documents, Turnhout, Brepols, 2003,372 p. Devant la recrudescence des études sur les ordres religieux médiévaux depuis cinquante ans, les auteurs ont jugé indispensable la réalisation d'un guide pour s'orienter dans la bibliographie pléthorique écrite à ce sujet. Il faudrait écrire le même type d'ouvrage pour les chanoines séculiers.

$4 \quad$ Le monde des chanoines (XI $I^{e}$-XIV siècles) (Cahiers de Fanjeaux, 24), Toulouse, Privat, 1989, 406 p.

$5 \quad$ Le monde des chanoines... [voir n. 4], p. 7-12. 
nécessité de consacrer un volume des Cahiers des Fanjeaux (qui en 1989 n'est encore qu'une « introduction ») à «la place tenue dans la vie religieuse du Languedoc, au coeur du Moyen Âge, par cette classe originale de clercs qu'on appelle chanoines ». Mais l'auteur s'intéresse d'emblée, poussé par l'examen plus particulier du cas languedocien, au monde des chanoines touchés par le mouvement de la réforme grégorienne et unifiés par le respect de la Règle d'Augustin en rappelant seulement «à côté d'eux, la masse des chanoines séculiers », ceux de type carolingien, les réformés de la Règle d'Aix. En cela, il illustre la déconsidération contemporaine de la réforme à l'endroit des chanoines séculiers, quand Nicolas II puis Urbain II appelaient de leurs voeux le retour à la vie commune pour des clercs considérés comme partout décadents. Ceux qui retrouvent le modèle de la vie apostolique primitive sont d'emblée et pour très longtemps placés au sommet dans la hiérarchie des saintetés, même pour les tâches pastorales alors que la synthèse du Père J. Avril $\left({ }^{6}\right)$ constate l'arrêt dès le $13^{\mathrm{e}} \mathrm{s}$. des régularisations de chapitres cathédraux car le mode de vie régulier était incompatible avec « les mutations de la fonction épiscopale, la gestion du temporel capitulaire, la nouvelle conception du canonicat devenu bénéfice ». Cela est interprété sous le seul mode de l'échec et s'ensuit un sombre tableau, peut-être caricatural, du chanoine séculier, a fortiori à la fin du moyen âge: simple clerc, membre d'une cour cardinalice ou pontificale, considérant le canonicat comme simple source de revenus et délaissant les obligations qui lui incombent. Ces conclusions, particulièrement sur le manque d'esprit de corps dans le monde canonial, sont en partie à revoir, en incluant vraiment les séculiers dans le monde canonial.

De manière générale, cette recherche sur les chanoines réguliers est très centrée sur le moyen âge central, ceux-ci connaissant une période dorée entre 1080 et 1150 puis une phase de stagnation voire de déclin, sauf pour les Prémontrés. Ils sont donc appréhendés dans le contexte précis de la réforme grégorienne $\left(^{7}\right)$ quand le retour à la vie régulière ne signifie rien moins que la survie du clergé tout entier au sein d'une société prédatrice. En cela, le discours réformateur marque l'historiographie au-delà même de cette époque, quand le danger n'est plus d'actualité mais il est inutile de rappeler que toute la spiritualité médiévale place le mépris du monde comme ultime voie vers le salut, même si grâce aux chanoines réguliers, le sacerdoce est enfin considéré comme un état de perfection possible et la vie active placée au même plan que la vie contemplative $\left({ }^{8}\right)$. Ce discours même, favorisé par l'appel sans cesse réitéré des contemporains à la réforme, est à nuancer en rappelant davantage la vraie résistance opposée par certains milieux comme les laïcs ou les chanoines séculiers qui refusent les régularisations d'églises au début du $12^{\mathrm{e}} \mathrm{s}$. Il est enfin quelquefois contredit par la lecture de sources un peu plus tardives qui témoignent de la gestion scrupuleuse des temporels ecclésiastiques par des chanoines séculiers, du partage des tâches entre une partie de résidents qui assurent les tâches quotidiennes et une autre partie vouée à une carrière de plus haut vol, absolument bénéfique au fonctionnement des institutions religieuses et surtout civiles, et enfin de la place laissée aux laïcs dans la liturgie et même par endroits, dans l'administration quotidienne de l'entretien du bâtiment.

La fin des années 1980 et le début des années 1990 ont connu la multiplication de travaux qui sont allés dans le sens de cette lecture nouvelle, voire de cette « réhabilitation » des séculiers, et particulièrement pour les chanoines des cathédrales. À côté de quelques monographies de chapitres, ces travaux se sont surtout orientés vers une perspective prosopographique $\left({ }^{9}\right)$. D'autres, de grande ampleur également, ont été dirigés par J.-Ch. Picard et Y. Esquieu $\left({ }^{10}\right)$ et ont fait des émules. Ils

$6 \quad$ Le monde des chanoines... [voir n. 4], p. 363-373.

$7 \quad C f$. comme exemple de travail récent sur les chanoines réguliers qui valorise fortement leur rôle dans la réforme de l'Église au $12^{\mathrm{e}}$ siècle: Des clercs au service de la réforme: études et documents sur les chanoines réguliers de la province de Rouen, sous la dir. de Mathieu ARNOUX (Bibliotheca victorina, 11), Turnhout, Brepols, 2000,404 p.

8 A. VAUCHEZ, La spiritualité du Moyen Âge occidental (VIII ${ }^{e}$-XIII siècle), Paris, Seuil, rééd. 1994,212 p., p. 87-91.

9 On doit saluer le rôle moteur d'Hélène Millet dans la publication continue depuis 1996 des volumes des Fasti Ecclesiae Gallicanae et la réflexion corrélative sur les évêques.

10 J.-Ch. PICARD (dir.), Les chanoines dans la ville. Recherches sur la topographie des quartiers canoniaux en France, Paris, De Boccard, 1994, 424 p. 
portent sur les quartiers canoniaux et témoignent de l'impact de la présence des chapitres dans le paysage urbain national.

Après cinquante ans d'études sur le monde canonial, le constat s'impose donc d'un retard pris par de comparables entreprises collectives à propos des chanoines de collégiales séculières. Oubliés des oubliés de l'histoire, pourrait-on dire, car ni revêtus du lustre de la régularité ni parés de la dignité cathédrale. Cet oubli est d'autant plus dommageable qu'ils sont très liés aux deux catégories précédentes, en premier lieu par le lien intrinsèque qui unit régularité et sécularité dans le monde canonial, les chanoines réguliers étant les «produits» des séculiers $\left({ }^{11}\right)$ et beaucoup d'établissements ayant connu les deux formes de vie au cours de leur histoire. Une étude parallèle et équilibrée des chanoines réguliers et séculiers gagnerait en authenticité historique d'autant qu'il y aurait concomitance des époques étudiées, le moyen âge central étant aussi la période d'apogée pour les chanoines de collégiales séculières. Du point de vue institutionnel ensuite, la filiation est souvent fondamentale entre cathédrales et collégiales séculières, même si leurs fonctions sont strictement différentes. Humainement, ce sont souvent les mêmes hommes qui peuplent ces deux types d'églises, voire les collégiales séculières et régulières. Cette lacune a été déplorée par la recherche ancienne et récente. Quelques jalons ont été posés dans l'historiographie mais ils viennent surtout des historiens du droit. Dans les ouvrages généraux d'histoire ecclésiastique qu'on leur doit, les collégiales séculières trouvent leur place dans la description des institutions de la Chrétienté mais dans une perspective résolument juridique $\left({ }^{12}\right)$. Ces institutions font l'objet d'un tableau très statique, vues surtout du point de vue des notions fondamentales du droit attachées à chaque maillon du rouage décrit. Il manque toute la dimension proprement historique, donnant l'épaisseur propre à la variété des cas, mais leurs auteurs soulignent clairement la difficulté d'appréhender le phénomène des collégiales dans sa globalité et sa continuité temporelle $\left({ }^{13}\right)$. La seule synthèse sur les églises collégiales souvent citée mais dont la diffusion est confidentielle car elle est restée inédite $\left({ }^{14}\right)$, est tout à fait issue de ce courant d'histoire juridique. Les ouvrages d'histoire ecclésiastique plus récents font les mêmes remarques $\left({ }^{15}\right)$ qui soulignent bien le lien des collégiales au moyen âge central avec

11 En témoigne la remarque de dom Becquet sur son travail dans son seul article synthétique consacré aux collégiales séculières: J. BECQUET, Collégiales et sanctuaires de chanoines séculiers en Limousin aux $X^{e}$-XII ${ }^{e}$ siècles, dans Bulletin de la Société archéologique et historique du Limousin, 103 (1976), p. 75-106 (75): «nos précédentes études sur les chanoines réguliers en Limousin auraient dû s'ouvrir par une revue de l'ordre canonial dans la même province, ordre dont le tronc devait garder l'épithète péjorative de séculier tandis que la branche augustinienne était dite régulière aux temps grégoriens. »

12 J. GAUDEMET dans le livre II chapitre III de: (dir.) Lot F. et Fawtier R., Histoire des institutions françaises au Moyen âge, publiée sous la direction de Ferdinand LOT et Robert FAWTIER. T. III: Institutions ecclésiastiques, par J.-Fr. Lemarignier, J. GAUdemet, G. Mollat, Paris, PUF, 1962, 502 p., consacre un passage aux « chapitres » p. 188-196, avec un premier temps sur le chapitre cathédral puis quatre pages sur la grande diversité des nombreuses collégiales due à leurs origines différentes. On y lit une description assez sèche du corps capitulaire avec ses dignités, ses modalités d'entrée, quelques jalons sur les régularisations, le rôle important dans l'encadrement paroissial, l'emprise de l'évêque et surtout des seigneurs dont les rois.

13 G. LE BRAS, Institutions ecclésiastiques de la Chrétienté médiévale (1130-1378) (Histoire de l'Église depuis les origines jusqu'à nos jours publiée sous la direction de Augustin FLICHE \& Victor MARTIN, 12), Paris, Bloud et Gay, 1964, 596 p., aborde la « coordination diocésaine » en replaçant les collégiales dans la liste des lieux de culte: paroisses, oratoires privés, églises de secours et chapelles publiques. Aux p. 425-426, il conclut sur le poids des collégiales dans le fonctionnement des diocèses, disant que la Chrétienté médiévale ne sera vraiment connue pour l'époque médiévale que «le jour où la géographie religieuse nous aura révélé la condition de tous les lieux de culte, et non seulement des paroisses ».

14 J.-H. LEGIER, Les églises collégiales en France, des origines au $X V^{e}$ siècle, thèse de la Faculté de droit de Paris, 1955.

15 Histoire du Christianisme des origines à nos jours, sous la dir. de Jean-Marie MAYEUR, Charles et Luce PIETRI, André VAucheZ, Marc Venard, T. V : Apogée de la papauté et expansion de la Chrétienté (1054-1274), sous la responsabilité de André Vauchez, Paris, Desclée, 1993, 973 p. On y lit onze pages sur les chanoines (p. 255-266) dues à M. Parisse, dont trois spécifiquement sur les chapitres autres que cathédraux. Ce discours est assez neuf sur les collégiales mais il signale qu'aucune étude globale n'existe sur le phénomène canonial, masqué par les travaux sur les seuls chapitres cathédraux au détriment des plus petites fondations: p. 259, « la chronologie de leurs fondations et leur dispersion géographique ont fait l'objet de peu d'études d'ensemble, alors que ces chapitres constituent pourtant le 
la mise en place des seigneuries et la croissance du réseau urbain. Ils donnent quelques éléments très éclairants en forme de pistes sur leurs origines, la personnalité des fondateurs, leur rôle de relais dans la politique religieuse menée par les évêques, les liens avec la géographie diocésaine, les fonctions variées des établissements (scolaire, caritative, pastorale, administrative) dont la moindre n'est pas de marquer le prestige de leurs bienfaiteurs. Ils rendent aussi justice au rôle avant tout sacerdotal du chanoine, même quand il n'est pas prêtre, à l'ouverture sur les fidèles par la liturgie canoniale, au rôle plus strictement paroissial de certaines églises, à leur puissance comme patrons et à leur poids dans la vie intellectuelle et les réseaux scolaires. Mais dès que l'on quitte cette époque dans la bibliographie générale $\left({ }^{16}\right)$, les collégiales ont à nouveau la portion congrue. Les travaux d'histoire urbaine $\left({ }^{17}\right)$ révèlent aussi ce manque quand elles ne prennent pas vraiment en compte les collégiales.

Ces perspectives existent pourtant dans la recherche internationale avec des travaux sur les collégiales séculières dans l'Empire (où elles constituent de très puissantes institutions sans commune mesure souvent avec les exemples de l'espace français), en Italie (où existent ces institutions très particulières que sont les pieve), dans les îles britanniques (les minsters), au Portugal, en Belgique. Le modèle du genre et proche de la démarche adoptée par notre équipe mais sans comparaison possible du fait de son ampleur, concerne la Suisse. L'Helvetia Sacra ou recension de tous les établissements religieux $\left({ }^{18}\right)$ des diocèses suisses du haut moyen âge au $20^{\mathrm{e}} \mathrm{s}$. est riche de fiches institutionnelles très détaillées pour chaque établissement (historique, description des fonds d'archives, bibliographie, notices des supérieurs de communautés qui proposent une large prosopographie). En France, les lacunes bibliographiques concernent non pas tant les monographies qui font l'objet de nombreux travaux universitaires et d'études ponctuelles dans les revues d'histoire régionale, qu'une synthèse de ce genre. Quelques publications montrent cependant la voie, anciennes et surtout récentes $\left({ }^{19}\right)$.

pendant des monastères » et en note 1: «d'une façon générale, une étude générale sur les chanoines et les chapitres manque cruellement », et encore p. 260: «or, des collégiales ont été fondées sans cesse de siècle en siècle dans des contextes différents et mériteraient une analyse attentive. »

$16 C f$. le t. VI de la même oeuvre: Un temps d'épreuves (1274-1449), Paris, Desclée-Fayard, 1990,945 p. Le chapitre 1 de la première partie par B. GUILLEMAIN sur «les cadres et les institutions de l'Église latine » contient un paragraphe de six lignes sur les collégiales séculières, p. 34, pour dire qu'elles ont proliféré et pointe quelques exemples isolés.

17 On peut citer par exemple de Ph. Lorentz Ph. et D. SANDron, Atlas de Paris au Moyen Âge, Paris, Éditions Parigramme, 2006, 237 p. Le chapitre 6 sur « la vie spirituelle » p. 110-167, détaille tout l'équipement religieux de la capitale: la cathédrale Notre-Dame, les paroisses, les cimetières, les ordres religieux mais il n'y a pas de rubrique spécifique sur les collégiales alors que Paris en abrite dix-huit à la fin du Moyen Âge (une page leur est consacrée, p. 139) et qu'elles ne sont pas toutes à chercher sous la rubrique «églises paroissiales » puisque certaines n'en sont pas. Les collégiales sont à nouveau évoquées dans la rubrique « les ordres canoniaux » mais celle-ci laisse une très large part à Ste-Geneviève et St-Victor montrant que le statut spécifique de la collégiale séculière n'a pas été repéré. Ces églises sont citées à d'autres titres dans le cours de l'ouvrage (paroisse, hôpital, etc.) mais cette dissémination est révélatrice de la sous- estimation du rôle des collégiales dans l'encadrement paroissial.

18 Pour les collégiales séculières: Le chiese collegiate della Svizzera italiana (Band II/1), Bâle, Schwabe, 1984, 178 p. et Die weltlichen Kollegiatstifte der deutsch- und französischsprachigen Schweiz (Band II/2), Berne, Schwabe, 1977, 686 p. On doit aussi signaler par l'un des auteurs du second volume deux articles de synthèse qui proposent une très vaste bibliographie rétrospective des p. 761 à 777 mais qui intègrent aussi les cathédrales: G.P. MARCHAL, Was war das Weltliche Kanonikerinstitut im Mittelalter? Dom- und Kollegiatstifte: eine Einführung und eine Neue Perspektive, dans R.H.E., 94/3-4, (1999), p. 761-807 ; 95/1 (2000), p. 7-53.

19 Dans le domaine de l'histoire de l'art, $c f$. la thèse de J. NoBLET, Collégiales castrales et Saintes-Chapelles à vocation funéraire dans le royaume de France (1450-1560), thèse de doctorat d'histoire de l'art, Université de Paris-IV, sous la direction de C. MignOT, 2 vol., 2005. En histoire, on peut retenir dans l'ordre chronologique: L. MUSSET, Recherches sur les communautés de clercs séculiers en Normandie au XI siècle, dans Bulletin de la Société des Antiquaires de Normandie, 55 (1959-1960), p. 5-38; P. CORBET, Les collégiales comtales de Champagne (v. 1150-v. 1230), Annales de l'Est, (1977/3), p. 195-241; J. NAZET, Les chapitres de chanoines séculiers en Hainaut du XII au début du XV $V^{e}$ siècle, Bruxelles, Académie royale de Belgique, 1993, 396 p.; Cl. BILlot, Les Saintes Chapelles royales et princières, Paris, Éditions du Patrimoine, 1998, 80 p.; V. TABBAGH, Un projet de recherches: les collégiales en Bourgogne au Moyen Âge, dans Annales de Bourgogne, 71 (1999), p. 99-117; H. NOIZET, Les basiliques martyriales au 


\section{Présentation de l'équipe et de ses travaux passés}

Ces travaux sont l'oeuvre de plusieurs des membres de l'équipe qui réalise depuis quelques années le répertoire annoncé. Elle est constituée d'une vingtaine de chercheurs de statuts et de spécialités diverses et a en charge à ce titre l'un des programmes de travail de l'axe VI, L'Église, corps social (XII $-X V I^{e}$ s.) de l'UMR 8589 (LAMOP-CNRS / Paris I) $\left({ }^{20}\right)$. Ce groupe a à son actif plusieurs réalisations communes en plus de la conception du répertoire. Il a participé à l'atelierséminaire organisé par Michelle Fournié de l'Université de Toulouse Le Mirail-FRAMESPA les 15 et 16 septembre 2000 à Carcassonne sur le thème des collégiales dans le Midi de la France $\left({ }^{21}\right)$. L'année suivante, ses membres ont organisé la troisième journée du colloque international « Histoire médiévale et christianisme » mis sur pied par le Groupement de recherche Gerson du CNRS à Orléans du 28 au 30 septembre 2001, avec pour base la collégiale St-Aignan d'Orléans $\left({ }^{22}\right)$. De même, l'équipe fut l'un des partenaires de la rencontre scientifique organisée les 12 et 13 juin 2004 avec l'UMR 5138 de l'Université de Lyon II $\left({ }^{23}\right)$ sur L'organisation de l'espace ecclésial, pour mener une réflexion commune entre historiens des textes et historiens du bâti. L'espace privilégié des discussions fut le choeur des édifices, collégiales, cathédrales et abbayes. Après une mise au point sur des questions de terminologie (choeur, chevet, abside, confession, sanctuaire), il fut question de la répartition et de l'affectation des espaces, particulièrement en matière liturgique, de la modification de ceux-ci quand une église est érigée en collégiale ou en cathédrale, des problèmes de franchissement et de circulation pour l'accès aux reliques ou encore pour accéder aux tombeaux souvent installées dans le choeur, et de la connaissance du mobilier qu'il abrite (stalles, luminaire) $\left({ }^{24}\right)$. Le groupe de travail a eu enfin comme dernier rendez-vous une journée d'études le 29 juin 2007 à l'Université de Limoges, en partenariat avec l'Équipe d'Accueil 3840 (CERHILIM) et ayant comme sujet les collégiales d'un grand centre-ouest envisagées comme enjeux de pouvoirs.

\section{Construction du répertoire}

Pour tenter une synthèse sur le monde canonial séculier, il a semblé à cette équipe qu'il était impossible de faire l'économie d'une recension complète des établissements qui furent une

$V I^{e}$ et au début du VII siècle, dans Revue d'histoire de l'Église de France, 89/219 (2001), p. 329-355; M. MAILLARDLUYPAERT, Papauté, clercs et läics. Le diocèse de Cambrai à l'épreuve du Grand Schisme d'Occident (1378-1417), Bruxelles, Publications des Facultés universitaires Saint-Louis, 2001, 647 p.; M. FoL, La création des collégiales dans le diocèse de Genève sous les pontificats de Clément VII et Benô̂t XIII (vers 1389-vers 1406). Des enjeux entre l'Église et l'État à l'échelle européenne?, dans La Savoie dans l'Europe. Actes du XXXVIII Congrès des Sociétés Savantes de Savoie, Moûtiers, 9-10 septembre 2000 (Mémoires et documents de l'Académie de la Val d'Isère. ns, 27), MoûtiersTarentaise, 2002, p. 41-79; B. MEIJns, L'ordre canonial dans le comté de Flandre depuis l'époque mérovingienne jusqu'à 1155. Typologie, chronologie et constantes de fondations et de réforme, dans R.H.E., 97/1 (2002), p. 5-58 (avec une bibliographie très complète); M. FouRnIE, Les collégiales dans le Midi de la France au Moyen Âge. Actes de l'atelier-séminaire des 15 et 16 septembre 2000 (Carcassonne), Carcassonne, UMR Framespa, GDR Salve, CVPM, 2003, 290 p.; Ch. MeRIAuX, Communautés de clercs et communautés de chanoines dans les diocèses d'Arras, Cambrai, Tournai et Thérouanne (VII ${ }^{e}-X I^{e}$ siècles), dans Frühformen von Stiftskirchen in Europa. Funktion und Wandel religiöser Gemeinschaften vom 6. bis zum Ende des 11. Jahrhunderts. Festgabe für Dieter Mertens zum 65. Geburtstag (Schriften zur Südwestdeutschen Landeskunde, 54), Leinfelden-Echterdingen, DRW-Verlag, 2005, p. 251-286; J. PERICARD, Ecclesia Bituricensis. Le diocèse de Bourges des origines à la réforme grégorienne, Clermont-Ferrand, L.G.D.J., 2006.

$20 \quad C f$. lamop.univ-paris1.fr

21 Les communications présentées lors de cette rencontre ont eu trait à l'histoire institutionnelle, archéologique et artistique de collégiales principalement situées dans le sud-ouest mais avec des perspectives comparatives grâce aux contributions portant sur les régions du nord et de l'est. Elles avaient surtout comme angle d'approche la problématique de l'intégration des établissements dans les diocèses et les cités: rapports avec les paroisses, les cathédrales, la géographie diocésaine, etc. Se reporter à la publication de ce volume mentionnée note 19.

22 Les exposés proposés portaient sur l'histoire du bâti et principalement de la crypte de St-Aignan, sur le culte des reliques, la liturgie chorale, le cloître et les chanoines de la collégiale orléanaise et d'autres exemples similaires.

23 Et particulièrement le groupe de recherche ACR « Morphogénèse de l'espace ecclésial » dirigé par Nicolas Reveyron et Anne Baud.

24 Le texte des communications sera disponible à la consultation sur le site internet qui abritera également le répertoire. 
collégiale séculière au cours de leur histoire. De là est née l'idée de construire un instrument de travail dans le même esprit que les anciennes entreprises d'inventaires $\left({ }^{25}\right)$ encore très commodes de nos jours, mais qui sera perfectible car toujours complété grâce à sa mise en ligne et aux techniques modernes de retouches permanentes.

Il a fallu pour cela se mettre d'accord sur une explicitation du terme contemporain de «collégiale », exercice effectivement périlleux $\left({ }^{26}\right)$ dans la mesure où il fallut réfléchir à partir de cas divers pour aboutir à une définition qui seule permit ensuite de cadrer les limites du recensement. L'outil réalisé sera d'autant plus vaste que la définition contemporaine de ce terme épineux excède les limites imparties par son usage dans les sources. On inclut en effet dans l'inventaire des établissements qui, à l'époque médiévale, ne se nomment pas collégiales ni leurs membres chanoines. Le mot « collégiale » apparaît tardivement dans la documentation alors que la réalité qu'il désigne pour nous existe déjà depuis longtemps et une fois qu'il est employé, il ne désigne pas, loin s'en faut, tous les établissements retenus comme conformes à la définition générale. La plus simple que l'on puisse proposer est de considérer comme collégiale tout établissement desservi par un groupe de clercs (deux au minimum) chantant ensemble l'office divin tant il apparaît évident que cette fonction liturgique est le seul dénominateur commun entre toutes les églises, et disposant d'une autonomie minimale, au moins physique, ce qui exclut les communautés de clercs dépendantes d'une autre institution: sous-collèges de chanoines d'un chapitre cathédral, méparts. Ces sociétés de prêtres $\left({ }^{27}\right)$, fondées beaucoup plus librement et spontanément que les collégiales, sans acte d'érection émanant d'un fondateur ou d'une autorité ecclésiastique, se distinguent d'elles par le fait qu'elles restent sous l'autorité du curé ou de la fabrique, quoique chantant parfois l'office en commun. La restriction aux collégiales séculières a ensuite conduit à une démarche inverse de celle qui a présidé pour une grande part dans l'historiographie canoniale, c.-à-d. que sont retenus les seuls établissements qui furent séculiers au cours de leur histoire et qu'ils sont « quittés » quand ils se transforment ou qu'ils se régularisent.

Cette définition fut rendue plus nécessaire encore par le caractère très interrégional du projet dont on va détailler les contours spatiaux et temporels. Les premiers incluent une France définie par les frontières de ses diocèses médiévaux, en retenant tous ceux dont une partie au moins des paroisses appartenaient au royaume au début du $16^{\mathrm{e}} \mathrm{s}$. ou à la France actuelle. De ce fait se trouvent incluses des régions encore peu connues à la différence de la Flandre, de la Champagne ou du domaine royal mis en lumière grâce à leurs collégiales seigneuriales de l'époque féodale dont le rôle politique fut éminent. Le répertoire inclut ensuite les établissements existant en 816 et se poursuit

25 Comme le «Beaunier-Besse »: Abbayes et prieurés de l'ancienne France, recueil historique des archevêchés, abbayes et prieurés de France, par dom BEAUNIER, nouvelle édition revue et complétée par les Bénédictins de Ligugé de 1905 à 1989 (qui propose pour chaque établissement une brève notice, la documentation manuscrite et imprimée, une bibliographie et une liste de personnages) ou encore le «dom Cottineau»: L.-H. CotTINEAU, Répertoire topobibliographique des abbayes et prieurés, Mâcon, 2 vol., 1935-1939, réimprimé par Brepols en 1996, qui ne s'intéresse qu'aux établissements réguliers, avec pour chacun le vocable, la localité ainsi qu'un état très sommaire des sources et de la bibliographie.

26 De manière parlante, les dictionnaires historiques peinent à donner une définition satisfaisante de la collégiale. Ils laissent une impression de définitions toujours partielles face à la diversité des cas, faute de synthèse faisant le point de ces aspects multiformes. Y échappe davantage l'époque moderne grâce à l'article Collégiales de Ph. LOUPES dans Dictionnaire de d'Ancien Régime: royaume de France, XVI $-X V I I I^{e}$ siècle, publ. sous la dir. de Lucien BELY, Paris, Presses universitaires de France, 2002, 1384 p., p. 285-286. L'auteur y donne une plus large part à la synthèse à partir de son propre travail sur le sud-ouest: répartition géographique des collégiales, lien privilégié entre collégiales et France rurale, inégale répartition dans les villes, relations entre collégiales et cathédrales, très grande diversité dans le détail des exemples sur la localisation, la richesse, la taille, le prestige.

27 Le colloque de la Société d'Histoire religieuse de la France (Les associations de prêtres en France du Moyen Âge à l'époque contemporaine. Actes du colloque organisé les 15, 16 et 17 septembre 2005 par la Société d'histoire religieuse de la France et le Centre d'histoire "Espaces et Cultures » (université Blaise Pascal), dans Revue d'histoire de l'Église de France, 93/230 (2007), 420 p., a récemment attiré l'attention sur elles ainsi que l'ouvrage de S. Gomis faisant suite à sa thèse: Les enfants prêtres des paroisses d'Auvergne, XVI ${ }^{e}$-XVIII siècles (Études sur le Massif Central), Clermont-Ferrand, Presses universitaires Blaise-Pascal, 2006. 
avec tous ceux qui furent créés jusqu'en 1530. La date de 816 a été arrêtée comme terminus a quo car alors la Règle d'Aix est généralisée à tous les établissements desservis par des clercs et particulièrement aux établissements non cathédraux dont les membres sont priés de se distinguer des moines et que l'on va désormais appeler chanoines dans la plupart des cas. Cette Règle propose une organisation à des formes de vie collective qui existent déjà depuis l'époque mérovingienne hors des cathédrales mais elle ne constitue pas forcément une réforme rendue nécessaire par un mouvement de décadence qui aurait suivi une faste période de fondations. Les églises qui existent avant 816 et qui n'ont pas perduré au-delà sont laissées de côté, faute de sources permettant de les bien connaître, mais elles sont peu nombreuses. La borne de 1530 s'explique par le souci de ne pas trop empiéter sur la période moderne mais aussi par celui de s'approcher de l'apogée numérique des collégiales repéré par Philippe Loupès $\left({ }^{28}\right)$ au milieu du $16^{\mathrm{e}} \mathrm{s}$. avec quelque cinq cents établissements dans le royaume de France d'alors.

En respectant ces limites, chaque collaborateur de l'équipe remplit une base de données pour un ou plusieurs diocèses. Cette base couvre aujourd'hui quatre-vingt seize diocèses $\left({ }^{29}\right)$ qui ont trouvé preneur et contient actuellement un total avoisinant les sept cents établissements recensés. Le chiffre sera probablement proche du millier quand tout l'espace imparti sera couvert. Pour ce faire, il faut avoir dépouillé un tronc commun de sources qui garantit que la recherche sur chaque collégiale a bénéficié d'une documentation à peu près comparable. Il s'agit des pouillés, des lettres pontificales publiées pour les $13^{\mathrm{e}}$ et $14^{\mathrm{e}}$ s., des comptes de décimes, des comptes rendus de visites pastorales, des Instrumenta de la Gallia christiana ainsi que des pièces justificatives publiées par la bibliographie existant sur le diocèse, des répertoires numériques des Archives départementales, de toute la bibliographie générale. À la différence du projet des Fasti Ecclesiae Gallicanae, le répertoire ne vise pas l'enquête prosopographique et son angle d'approche est bien plutôt institutionnel. Les collégiales y sont appréhendées comme corps capitulaires mais également comme instances plus larges ancrées dans un environnement. On pourra en juger, les champs de la base de données renseignent surtout sur le chapitre mais aussi sur le bâtiment, son insertion dans la géographie diocésaine, humaine et ecclésiastique, et donc sur la collégiale stricto sensu d'où le libellé retenu pour le répertoire. Ce terme de «collégiales » a été préféré là à celui de "chapitre » car dans la terminologie courante, la collégiale renvoie plutôt à un établissement séculier que régulier dans la mesure où les établissements réguliers sont également appelés abbayes, prieurés par rapprochement avec le monde monastique. Les champs de la base sont assez sommaires mais cela se justifie par l'étendue du travail et le souci d'élaborer un masque de saisie pouvant s'adapter à tous les cas rencontrés et s'affiner au fur et à mesure du remplissage. Ils sont les suivants:

\footnotetext{
- date de fondation (soit la date d'érection de la collégiale ou à défaut la date d'attestation à laquelle on a la première mention d'une communauté de chanoines, avec cet impératif de repérer ce terme dans les sources, ce qui conduit à postdater certaines d'entre elles mais c'est le prix à payer pour obtenir un critère commun de datation et respecter ainsi une rigueur scientifique),

- - localité (localité ancienne, commune actuelle, code INSEE, canton, département, pays),

- - vocable,

- - province ecclésiastique,

- - diocèse,

- $\quad$ - fondateur,
}

28 Dans son article Collégiales [voir n. 26], p. 285-286.

29 Il s'agit de: Agen, Aire, Albi, Alet, Amiens, Angers, Arras, Auch, Autun, Auxerre, Avignon, Avranches, Bayeux, Bayonne, Bazas, Beauvais, Belley, Besançon, Béziers, Bordeaux, Bourges, Cahors, Cambrai, Carcassonne, Carpentras, Castres, Cavaillon, Chalon, Chambéry, Chartres, Clermont, Condom, Coutances, Dax, Die, Elne, Evreux, Gap, Genève, Grenoble, Langres, Laon, Lausanne, Lavaur, Lectoure, Le Puy, Lescar, Limoges, Lisieux, Lombez, Luçon, Lyon, Mâcon, Maguelonne, Maillezais, Meaux, Mirepoix, Montauban, Nevers, Noyon, Oloron, Orange, Orléans, Moutiers-en-Tarentaise, Pamiers, Paris, Périgueux, Poitiers, Rieux, Rodez, Rouen, St-Bertrand de Comminges, St-Flour, St-Jean de Maurienne, St-Lizier de Couserans, St-Papoul, Saintes, Sarlat, Sées, Senlis, Sens, Sion, Soissons, Tarbes, Thérouanne, Toulouse, Tournai, Tours, Troyes, Tulle, Vabres, Vaison, Valence, Vienne, Viviers, Uzès. De grands espaces restent encore à couvrir: Alsace, Bretagne, Lorraine, Provence. 
- - état antérieur (soit l'institution préexistante ce qui permet de remettre dans une perspective historique les nombreuses institutions préexistantes devenues chapitres, avec la date de création de la première institution),

- $\quad$ - nombre de chanoines (accompagné de la date d'attestation pour prendre en compte les évolutions numériques, à défaut nombre de bénéfices total du chapitre),

- patron,

- $\quad$ collateur,

- $\quad$ - date de disparition (ou de transformation avec mention de la nouvelle institution),

- $\quad$ - remarques (points incertains ou précisions des autres champs comme les dignitaires du chapitre par exemple),

- $\quad$ - documents (corpus iconographique: plans, photographies, etc.),

- - bibliographie (mention des sources manuscrites avec renvoi aux cotes d'archives, sources imprimées d'importance comme les cartulaires et orientation bibliographique qui vise l'essentiel par la citation de cinq titres dont des monographies, des études récentes riches d'une bibliographie plus ancienne, des études architecturales ou d'histoire de l'art quand elles sont les seules à exister sur le sujet).

La base formant répertoire utilise le format XML qui permettra la présentation d'une fiche synthétique pour chaque collégiale et surtout une interrogation par l'utilisateur sur toutes les informations contenues dans cet outil. Elle offrira cette facilité commune à d'autres outils de ce genre de rechercher des réponses ciblées sur une localisation, une titulature, une date de fondation, de disparition, de transformation. Elle sera prochainement disponible sur le site internet en cours de construction et abrité par le portail actuel du LAMOP. Elle proposera à la disposition des chercheurs d'autres instruments de travail: une introduction méthodologique et scientifique au répertoire, une cartographie de localisation des établissements et une bibliographie sur les collégiales de l'espace français au moyen âge qui visera peu à peu à l'exhaustivité. L'équipe prévoit également une série de synthèses régionales sur les collégiales médiévales et modernes dont la première est constituée par la publication de la journée d'études de Limoges en juin 2007 sur les collégiales du Limousin, du Périgord, du Berry, du Rouergue et de l'Auvergne. D'autres devraient suivre sur le Languedoc puis sur un grand quart nord-est, ces synthèses permettant d'aborder des problématiques plus complexes que le répertoire.

\section{Intérêts scientifiques de cet outil de travail}

Conçu comme un indispensable prélude à toute étude de synthèse sur les collégiales, cet outil devra permettre la constitution d'une « géo-chrono-typologie » des collégiales en proposant un travail collectif qui remette en lumière de très petits ou très éphémères établissements souvent oubliés par les études qui ne citent que les églises prestigieuses et bien documentées. Cette étude quantifiée du mouvement chronologique de création des collégiales sur une large échelle géographique sera à replacer en premier lieu dans le contexte de la création des autres réseaux d'encadrement ecclésiastique (paroisses, monastères, abbayes de chanoines réguliers, hôpitaux, couvents mendiants). Mais elle permettra aussi une exploitation comparée des données du répertoire sur l'institution capitulaire proprement dite et sur ses formes évolutives. D'autres questions méritent l'attention des chercheurs, celle du lien entre collégiales et réseau urbain et plus encore celle des rapports entre collégiales et pèlerinages, à toutes époques, depuis les basiliques périurbaines fondées sur des corps saints prestigieux comme St-Quentin ou St-Martin de Tours, jusqu'aux fondations tardives parfois dotées par leurs bienfaiteurs de reliques insignes. Le moindre mérite de cet instrument ne sera pas de permettre aux spécialistes de l'histoire d'un diocèse ou d'une région un débroussaillage institutionnel sur le statut exact de ces églises et sa durée au long d'une histoire souvent mouvementée, ce qui évitera approximations voire anachronismes. Les études d'histoire de l'art et d'archéologie, nombreuses sur les églises collégiales, pourront y trouver le support d'informations institutionnelles faciles à reprendre. De même, il devrait offrir la possibilité de démêler les liens complexes (souvent fondés sur la collation des bénéfices) qui existent entre établissements ecclésiastiques d'un ou de plusieurs diocèses puisqu'il contient la mention du patron et du collateur des prébendes de la collégiale avec leurs dates d'attestation et les bénéfices détenus par eux. Les chanoines particulièrement présents dans l'histoire des diocèses pourront être replacés 
dans leur environnement institutionnel, particulièrement les dignitaires des chapitres. Grâce à l'inventaire de la documentation, seront mise en lumière les sources parmi les mieux conservées sur la composition et le rôle du clergé séculier, surtout dans ses fonctions d'encadrement pastoral. D'un point de vue plus spirituel, il faudrait tenter une synthèse sur le développement de la sécularité face au monde chatoyant des réguliers, permise par la mise en forme systématique pour chaque établissement des traces de la pression exercée par le modèle régulier à partir de la réforme grégorienne, visibles dans la transformation des établissements. Par les titulatures des collégiales que donne le répertoire, sera éclairé le culte de saints très locaux dont elles ont pris le nom et dont elles vénèrent les reliques, quelquefois bien plus que les cathédrales ou les établissements monastiques. Pour l'historien de la société enfin, il sera possible de reconstituer facilement le volet religieux de la politique d'implantation territoriale de familles seigneuriales et / ou d'évêques grâce à la mention de l'identité du fondateur de la collégiale et de repérer de manifestes alliances entre autorités laïques et ecclésiastiques, d'autant que la fondation de collégiales est une démarche spirituelle fréquente, au moins autant que les fondations monastiques dans la mesure où ces églises ont toujours été préférentiellement ouvertes aux laïcs.

\section{Les acquis actuels: une esquisse de typologie des collégiales séculières pour la France médiévale}

Les collégiales séculières de France ont continué d'exister et même d'être créées tout au long de la période médiévale comme modèle d'organisation ecclésiastique sans cesse revisité par leurs fondateurs, présentant des modalités très différentes selon les époques et une grande diversité régionale. Au début du travail de recension, on s'attendait à distinguer clairement des vagues de fondations de collégiales privilégiant telle époque puis des moments de désaffection, peut-être à l'aune du mouvement des fondations monastiques. En fait, cela n'est pas aussi manifeste, la réalité étant bien plutôt un mouvement de fondations continues. Certes, des pics apparaissent du point de vue du nombre des établissements fondés mais il n'y a pas une décennie au cours de ces sept siècles qui ne voie la création d'une collégiale ou en tout cas son apparition dans la documentation pour les époques les plus anciennes, entre 800 et 1000. La période d'engouement est très évidemment le $11^{\mathrm{e}} \mathrm{s}$. Mais les décennies suivantes sont aussi bien pourvues, illustrant ce caractère continu du phénomène, même s'il suit une tendance décroissante. Il ne faut pas oublier non plus la question de la pérennité de ces églises car beaucoup sont transformées ou disparaissent, avant même la fin du moyen âge. Beaucoup des créations du $11^{\mathrm{e}}$ s. ont été ensuite régularisées, alors que les collégiales fondées ensuite ont souvent perduré jusqu'au $18^{\mathrm{e}} \mathrm{s}$. Actuellement, on peut établir que $40 \%$ des églises répertoriées pour l'époque médiévale ont vécu de manière sûre au delà de 1530. Géographiquement, elles existent partout dans l'espace défini, ce qui fait aussi l'intérêt du sujet. Leur répartition y est assez homogène même si se dégagent des zones de densité plus forte: Flandre, Île-de-France, Bourgogne, Berry, Limousin, au détriment d'un quart sud-est plus pauvre. Pour dresser une typologie des ces églises, il est nécessaire de reprendre l'histoire de leur mise en place tout au long du moyen âge et d'examiner le contexte de leur fondation.

Avant 816 , on ne parle bien sûr pas encore de collégiales ni d'ailleurs de chanoines pour les autres églises que les cathédrales mais de basiliques, desservies par des clerici qui ne sont pas des moines même si leur supérieur est souvent nommé abbas. Ce ne sont pas pour la plupart (et le flou de la terminologie a encouragé cette interprétation) des communautés monastiques décadentes devenues communautés de clercs. Ceux-ci sont membres du presbyterium entourant l'évêque de la cité qui leur délègue partie de sa juridiction pour desservir les basiliques. Ces communautés sont d'ailleurs souvent organisées en réseaux autour de l'église-mère avec laquelle elles entretiennent des liens institutionnels forts $\left({ }^{30}\right)$. Leurs abbés voire la communauté tout entière participent par exemple à l'élection de l'évêque. Ces particularités déroutent souvent l'historien quand il les saisit plus tard

30 C'est le cas à Lyon entre St-Jean et plusieurs communautés très intimement reliées à elle: St-Paul attestée vers 810 et St-Nizier, St-Just et St-Irénée attestées vers 830. 
dans la chronologie. Elles sont aussi associées par des liens liturgiques, le presybterium, futur chapitre cathédral, se rendant en station dans telle basilique pour célèbrer l'office à certains moments de l'année. Leur organisation institutionnelle est très mal connue, elles se nomment monasterium, coenobium, congregatio, conjuratio même, ce qui atteste la pratique de la vie commune. Elles assurent la desserte de basiliques funéraires ou cimétériales, souvent fondées sur le lieu d'inhumation d'un saint ermite ou saint évêque mérovingien dont les reliques suscitent la création de pèlerinages, et elles abritent aussi souvent un hôpital qui accueille pauvres et malades. Ces basiliques à collèges sont plutôt périurbaines qu'implantées à l'intérieur de la cité qui leur doit la création de faubourgs lentement peuplés aux époques mérovingienne et carolingienne (d'où le terme employé également à leur endroit de basiliques suburbaines). Elles existent aussi en campagne, souvent mais pas forcément dans les chefs-lieux de vici, et là aussi, elles sont à l'origine de nouveaux lieux de peuplement $\left({ }^{31}\right)$. Ces basiliques deviennent des centres paroissiaux au fur et à mesure de la mise en place du maillage paroissial et du démembrement de l'unique paroisse originelle. Elles constituent souvent des relais essentiels de l'autorité de l'église-mère au sein du diocèse et dans la cité, d'autant plus quand elles sont situées au sein de fiefs épiscopaux: on les nomme d'ailleurs explicitement «filles de l'évêque $\left({ }^{32}\right)$ » dans le courant du moyen âge. L'encadrement paroissial est également assumé par des communautés d'un autre type, les communautés de clercs (souvent d'anciens moines) constituées auprès des monastères mixtes ou des monastères de femmes fondés à l'époque mérovingienne, afin de célébrer l'Eucharistie et de délivrer les sacrements aux moniales. Ces collèges ont même quelquefois supplanté le monastère féminin au $9^{\mathrm{e}}$ ou $10^{\mathrm{e}}$ siècle et souvent, ils ont reçu la charge de la paroisse créée dans le bourg monastique $\left(^{33}\right)$.

En 816, ces communautés sont priées d'adopter la Règle d'Aix et dès lors, le terme canonicus peut renvoyer aux clercs (qu'on aurait bien tort de qualifier alors de chanoines séculiers) qui desservent ces églises qui, elles, ne sont pas encore appelées collégiales. Dans les faits, il est très difficile de dire pour chacune si elles ont appliqué la regula canonicorum et n'en sont pas restées à des usages locaux subsistants. Les sources indiquent tout de même que certaines d'entre elles $\left({ }^{34}\right)$, souvent sous l'égide de l'évêque, ont procédé à la division de leur patrimoine en prébendes et ont fait construire des bâtiments communs $\left({ }^{35}\right)$. Beaucoup de ces églises subissent des destructions à la fin de l'époque carolingienne et se trouvent désorganisées. Elles disparaissent des sources au $9^{\mathrm{e}}$ s.et on ne sait ce qu'elles deviennent $\left({ }^{36}\right)$. D'autres en revanche doivent leur création au contexte troublé d'alors, particulièrement quand elles sont fondées par une communauté en fuite devant les invasions et soucieuse de protéger ses reliques $\left({ }^{37}\right)$. Les exemples les plus parlants sont les collégiales créées sur les lieux de refuge des chanoines de St-Martin de Tours, par exemple StMartin de Chablis dans le diocèse de Langres, attestée vers 872 et appelée prévôté, ancien monastère donné par Charles le Chauve aux chanoines tourangeaux refugiés là en 867 . Les dignitaires de cette époque sont souvent appelés prévôts. Ce terme, qui apparaît à l'époque carolingienne et que l'on retrouve dans les cathédrales, est une preuve des liens maintenus et des imitations entre les deux types d'établissements.

31 Comme à St-Cloud, fondée par le petit-fils de Clovis dans le diocèse de Paris ou encore à St-Martin de Brive, sur le lieu du martyr de Martin l'Espagnol au $5^{\mathrm{e}}$ s., où les deux premiers bâtiments successifs sont l'oeuvre des évêques de Limoges dans le courant du $6^{\mathrm{e}} \mathrm{s}$.

32 Tel est le nom de St-Germain l'Auxerrois fondée en plein fief épiscopal à Paris au $7^{\mathrm{e}}$ s. sur les reliques de l'évêque mérovingien Landry.

33 Dans le diocèse de Meaux, un collège de clercs attesté en 842 a pour fonction de desservir le monastère NotreDame fondé à Faremoutiers par Ste Fare au milieu du $7^{\mathrm{e}} \mathrm{s}$. Il a aussi la charge de la proche paroisse St-Sulpice.

34 Soit d'anciens monastères passés à l'ordo canonicus, soit des communautés scindées en deux après 816 , l'une canoniale, l'autre monastique, comme cela est le cas à Sithiu dans le diocèse de Thérouanne où deux communautés sont clairement distinguées en 820: 40 moines à St-Bertin et 30 chanoines à St-Omer.

35 C'est peut-être le cas à St-Marcel, dans un faubourg de la rive gauche à Paris, fondée au $5^{\mathrm{e}}$ s. sur les reliques de l'évêque éponyme et manifestement réorganisée par l'évêque Ingelvin vers 880.

36 Particulièrement dans les diocèses de Tournai, Cambrai, Thérouanne.

37 Cela est très vrai des reliques de saints de l'ouest du royaume, par exemple celles de Ste Opportune, originaire du département actuel de l'Orne, transportées et fixées à Paris vers 879 et à l'origine de la création d'une petite collégiale de la rive droite. 
$\mathrm{Au}$ début du moyen âge central, soit au $11^{\mathrm{e}}$ s., apparaît le terme de collégiale. Cela est à mettre en relation avec l'explosion numérique de ces églises qui connaissent alors leur âge d'or. Suit un mouvement de fondation important dans la seconde moitié du $12^{\mathrm{e}}$ s., qui correspond à un essor général du clergé séculier. Les instigateurs de ce phénomène sont faciles à repérer d'autant que la documentation commence alors à être bien plus fournie. Cela part des princes, en premier lieu la maison de Champagne, mais sans doute aussi celles de Flandre et d'Artois et le relais est pris entre 1180 et 1220 par les évêques, au moins dans une région qui englobe les diocèses de Nevers, Auxerre, Sens, Meaux et sans doute aussi Troyes. Pour ce milieu épiscopal, la fondation de collégiales est un élément important dans une stratégie pastorale de développement du service divin et d'encadrement des fidèles. Il est d'ailleurs notable que le lieu d'implantation des collégiales corresponde assez souvent aux chefs-lieux des subdivisions de la géographie diocésaine, archidiaconés, archiprêtrés, doyennés ruraux, qui sont créés au $11^{\mathrm{e}}$ et surtout au $12^{\mathrm{e}} \mathrm{s}$. ${ }^{38}$ ). Les premiers fondateurs de collégiales créées ex nihilo sont donc les seigneurs laïcs qui accaparent le pouvoir en ces temps féodaux, constructeurs de châteaux et qui fondent en même temps ou plus tard, dans la mesure de leurs moyens, une collégiale, quelquefois ancienne chapelle, qu'on a qualifiée de collégiale castrale. Ces petites ou grandes structures $\left({ }^{39}\right)$ sont à replacer dans le contexte de la restitution des dîmes par des seigneurs placés à toutes les échelles, de la petite seigneurie au duché. Elles demandent tout de même un minimum de dotation pour constituer la mense, divisée assez rapidement ensuite en prébendes. Quand elles sont fondées sur le lieu d'un castrum, d'un marché et donc d'un bourg, elles participent à l'encadrement religieux de la croissance urbaine en devenant aussi églises paroissiales. En Bourgogne néanmoins, la fonction paroissiale, ou plus exactement sacramentelle, de ces églises paraît faible voire marginale car elles servent d'abord à fournir des revenus aux membres des familles seigneuriales entrés dans la cléricature. De fait, leur fonction principale est de constituer le volet religieux d'une politique de domination territoriale. De manière caractéristique, les collégiales sont nombreuses quand les lignages de la région sont puissants et surtout rivaux $\left({ }^{40}\right)$. Dans le comté de Flandre, il semble d'ailleurs que le seigneur assoie davantage son autorité grâce à la création d'une collégiale dont il a le patronage des bénéfices et grâce au système de l'avouerie. Là et ailleurs, les chanoines de ces églises constituent aussi un réservoir de personnel pour les chancelleries comtales et ducales. Cette politique de prestige est encore plus nette quand les collégiales sont aussi fondées pour abriter des reliques ramenées de croisade par les seigneurs fondateurs $\left({ }^{41}\right)$ et qui donnent à ces églises des titulatures très différentes de celles plus anciennes, qui portent le nom de saints mérovingiens. Appartiennent à cette souscatégorie les saintes chapelles dont le prototype est la Ste-Chapelle du Palais à Paris fondée au milieu du $13^{\mathrm{e}} \mathrm{s}$. par Louis IX pour honorer les reliques de la Passion. Cette « relégation » à un rôle strictement liturgique et surtout la complète domination qu'exercent sur elles les fondateurs laïques et leurs descendants, sont manifestes par leur titre même de saintes chapelles aux membres desquelles il sera longtemps dénié le titre de chanoines, de même que celui de collégiales à leurs églises. Quant aux évêques et chapitres cathédraux, ils réorganisent à cette époque d'anciennes communautés, voire d'anciens monastères, quelquefois seulement érigés en vrais chapitres avec dotation de statuts, organisation du temporel, transformations de dignités dans le courant du $11^{\mathrm{e}} \mathrm{s}$. Par ailleurs, beaucoup de ces institutions restent attachées aux usages carolingiens et continuent de suivre la Règle d'Aix qu'elles vont seulement abandonner peu à peu dans le courant du siècle. Le but de cette réorganisation est-il très exactement le même quand son instigateur est l'évêque ou quand il s'agit du chapitre cathédral ? Cela reste à déterminer encore $\left({ }^{42}\right)$. De manière générale, avec

38 Cela se vérifie dans les diocèses de la région parisienne, de Savoie, de Languedoc, du Limousin.

39 Puisque certaines ont deux chanoines quand St-Etienne de Troyes, fondée par Henri le Libéral en 1157 , compte soixante-douze prébendes et neuf dignités.

40 Par exemple dans le diocèse de Bourges avec la maison des Capétiens, celle des comtes de Blois et celle des comtes d'Anjou.

41 À Luzarches dans le diocèse de Paris, le comte Jean de Beaumont dépose dans la collégiale attestée vers 1183 des reliques de Côme et de Damien qu'il a ramenées de son voyage en passant par Rome.

42 Mais quand le chapitre cathédral de Paris érige en collégiales ses trois « filles », St-Merry sur la rive droite vers 1005 et St-Benoît le Bétourné ainsi que St-Etienne des Grés sur la rive gauche vers 1050, il leur confère le titre 
l'apparition de nouvelles paroisses urbaines entraînée par l'essor démographique, les églises collégiales voient leur territoire originel se démembrer et les chapitres obtiennent souvent le patronage des nouvelles cures et une large partie des droits paroissiaux. Les évêques président d'ailleurs au règlement de ce partage des revenus. Les collégiales sont dès cette époque des maillons importants de la hiérarchie ecclésiastique d'une ville, quitte à « réabsorber » ces cures dans la mense capitulaire à la fin du moyen âge. Quand la localité n'est pas chef-lieu de diocèse, la collégiale a fréquemment la main mise sur tout ou partie du réseau paroissial $\left({ }^{43}\right)$. De la même manière, en milieu rural, les évêques sont les fondateurs de collégiales au sein de paroisses anciennes afin d'en consolider la desserte $\left({ }^{44}\right)$. Certains établissements du $12^{\mathrm{e}} \mathrm{s}$. sont aussi le résultat de la volonté de certains groupes informels de chapelains groupés autour de leur curé, de s'organiser en se dotant de statuts. Ce souhait est souvent relayé par la politique épiscopale mais il s'agit bien d'un mouvement d'auto-promotion qui vise à créer un corps gérant et répartissant les revenus provenant des nombreux dons faits par les fidèles pour la célébration des obits $\left({ }^{45}\right)$.

Dans le courant du $13^{\mathrm{e}}$ s. et surtout en ville, d'autres créations sont le fait de bourgeois riches, ou encore de nobles, qui envisagent aussi leur fondation comme le support d'autres activités que des activités seulement liturgiques. Il s'agit de chapitres créés pour la gestion d'un collège dans les villes universitaires ou encore pour celle d'un hôpital $\left({ }^{46}\right)$. Dès cette époque, et il faut y insister, ces églises ont joué un rôle fondamental comme pôle de développement de la prière pour les morts, en particulier celles établies dans une petite ville, pour des milieux modestes et pour les paysans des villages environnants qui ne fondent pas d'anniversaires chez les réguliers et encore moins dans les cathédrales, pas encore non plus dans les paroisses. On s'en aperçoit lorsqu'ont été conservés des livres d'anniversaires ou des actes royaux d'amortissement. Grâce à elles , des gens modestes ont pu accéder à la mémoire spirituelle. Beaucoup de ces églises filles du moyen âge central ont cependant été transformées ou ont tout simplement disparu, faute de ressources. La principale transformation qui les affecte est la régularisation, soit en prieuré bénédictin ou clunisien soit, plus logiquement, en collégiale régulière suivant la Règle de $\mathrm{S}$. Augustin quand le souci de la discipline intérieure a conduit le fondateur ou l'évêque du lieu à suivre les chemins de la réforme $\left({ }^{47}\right)$.

À la fin du moyen âge, les fondations de collégiales séculières se poursuivent et endossent peu ou prou les mêmes fonctions que celles déjà entrevues. Le $14^{\mathrm{e}} \mathrm{s}$. se distingue avec une vague de fondations cardinalices et pontificales sous la papauté d'Avignon. Ce sont souvent des fondations obituaires de prestige dans les diocèses d'origine des prélats, la collégiale étant d'abord nécropole et assumant une fonction liturgique $\left({ }^{48}\right)$. En témoigne la construction fréquente d'une église de grande ampleur souvent pourvue d'un cloître. Le caractère ostentatoire de ces établissements se lit nettement dans la qualité architecturale des bâtiments. Mais certaines ont de manière plus complexe une place fondamentale dans l'organisation de la vie citadine $\left({ }^{49}\right)$, ont encore un rôle à jouer dans le

d'églises cardinales, comme autant de pôles structurants de la réorganisation paroissiale de la ville.

43 Le chapitre St-Quentin à St-Quentin en Vermandois dans le diocèse de Noyon, doté de soixante-douze prébendes, est ainsi collateur de toutes les paroisses de la ville démembrées en 1214 de l'unique paroisse originelle dont le centre était la collégiale.

44 Simon, évêque de Meaux, procède ainsi en 1189 à l'érection en collégiale de l'église paroissiale St-Germain l'Auxerrois d'Oissery, en justifiant son geste dans son acte de fondation par le fait qu'« il est préférable pour une église d'être desservie par plusieurs clercs que par un seul ».

45 Cela est manifeste à St-Léger de Tannay en 1201 dans le diocèse de Nevers, là où le clergé du lieu instaure la collégiale ou encore à Bénévent dans les années 1070 avec intervention directe du chapitre cathédral de Limoges.

46 La collégiale Ste-Madeleine d'Aigueperse au diocèse d'Autun, créée en 1288 par Hugues d'Arcy, seigneur du lieu et évêque, reçoit la gouvernance de plusieurs hôpitaux.

47 Comme par exemple à Bénévent, devenue Bénévent-l'Abbaye dans le diocèse de Limoges après sa régularisation augustinienne vers 1095 .

48 La collégiale de La Chapelle-Taillefert est ainsi fondée en 1311 par le cardinal du même nom dans le diocèse de Limoges ou encore St-Germain les Belles en 1384 par les exécuteurs testamentaires d'Hugues Roger, frère de Clément VI.

49 En Avignon, l'église paroissiale St-Agricol est érigée en collégiale par Jean XXII en 1321 et elle abrite les réunions des instances consulaires de la cité. St-Didier, autre paroisse érigée en collégiale par le cardinal Bertrand de Déaut en 1359, fait de même pour les assemblées des membres de l'Université d'Avignon. 
renforcement de l'encadrement paroissial $\left({ }^{50}\right)$ ou reçoivent pour claire mission de contrebalancer le pouvoir du chapitre cathédral dans les nouveaux diocèses créés à partir de $1317\left({ }^{51}\right)$. Aux $14^{\mathrm{e}}$ et surtout $15^{\mathrm{e}}$ s., de petits seigneurs et surtout d'autres de plus grande envergure continuent de fonder des collégiales. Les évêques se comportent d'ailleurs de la même façon dans leur résidence secondaire ou dans leurs fiefs $\left({ }^{52}\right)$. Ces églises ont encore un rôle de prestige et certaines assortissent même la reconstruction d'un château au coeur des possessions seigneuriales pour en marquer la puissance, notamment de la part des serviteurs de la Couronne en Val de Loire. Leurs collégiales sont des nécropoles familiales. D'autres, appartenant à la lignée de S. Louis, fondent des saintes chapelles soulignant ainsi leur rattachement à une dynastie insigne $\left({ }^{53}\right)$. Le dernier cas est représenté par des collégiales étant le fruit de la promotion de ces groupes de prêtres filleuls alors très nombreux qui souhaitent acquérir une entité juridique. Elles sont quelquefois soutenues par les communautés d'habitants à la campagne ou en ville en vertu de leur désir de voir le service divin rehaussé dans leur église, et dans le sud-ouest $\left({ }^{54}\right)$, elles sont même promues par les consuls des cités qui sont patrons de ces collégiales nouvelles, la dimension liturgique et sacramentelle de ces collèges n'ayant pas disparu. On y développe d'ailleurs les cultes civiques autour de reliques propres à l'identité urbaine. Ailleurs, ces transformations institutionnelles suscitent des réticences de la part des fidèles $\left({ }^{55}\right.$ ) car elles donnent plus d'autonomie au corps sacerdotal alors que les laïcs contrôlaient assez étroitement les méparts.

À ce stade de la synthèse, on peut dégager quelques grandes caractéristiques propres aux collégiales médiévales de l'espace français qui sont autant de problématiques à développer encore. On aura remarqué les éléments qui participent de leur définition: leur rôle fondamental de lieux de prière, de récitation de l'office divin et souvent de culte de reliques, voire de pèlerinages ; leur importance dans l'organisation du clergé diocésain, avec ou sans règle ; leur place dans l'encadrement pastoral des fidèles laïques ou des moniales ; leur fonction de points d'ancrage de l'autorité seigneuriale, soit laïque soit ecclésiastique. Elles auront en tout cas illustré la force gardée par le modèle communautaire au sein d'un clergé séculier qui emprunta la voie étroite de ceux qui, fidèles à leur statut de clercs, n'ont pas renoncé au monde mais qui ayant entretenu des relations décomplexées avec les biens terrestres, ont eu le mérite d'aller à la rencontre des difficultés et des embûches que cela représentait pour l'accès à la sainteté. Ces choix, au coeur même des rapports entre spirituel et temporel, caractérisent les chanoines séculiers des collégiales. Par l'étude des caractéristiques de tous leurs chapitres et de toutes leurs églises, on espère appréhender mieux l'essence et la pertinence de leur mode de vie.

Anne MASSONI

Université de Limoges

50 Par exemple dans les diocèses savoyards, avec les collégiales fondées pendant le Schisme entre autres par Clément VII comme Notre-Dame de Liesse à Annecy en 1397 dans le diocèse de Genève.

51 Les collégiales fondées par Jean XXII dans le sud-ouest comme St-Etienne du Tescou à Montauban en 1318 participent même à l'élection de l'évêque, reproduisant ainsi des structures propres aux époques hautes.

52 Pierre d'Amboise, évêque de Poitiers, fonde ainsi une collégiale dans son château de Dissay entre 1481 et 1487.

53 Elles sont assez nombreuses au tournant des $15^{\mathrm{e}}$ et $16^{\mathrm{e}}$ s.: St-Louis d'Aigueperse fondée en 1475 par Louis de Montpensier ou la Ste-Chapelle de Riom en 1489 par Pierre de Beaujeu et Anne de France, toutes deux dans le diocèse de Clermont.

54 En 1447, à Villefranche de Rouergue, ce sont les consuls et les prêtres obituaires qui poussent à l'érection de la collégiale Notre-Dame.

55 Comme à Chaumont-en-Bassigny ou à Dijon dans le diocèse de Langres où deux collégiales St-Jean-Baptiste sont fondées à partir de méparts en 1474 et 1475. 